\title{
Simulations of viscoelastic droplet deformation through a microfluidic contraction
}

\author{
D. J. E. Harvie ${ }^{1}$, M. R. Davidson ${ }^{1}$ \& J. J. Cooper-White ${ }^{2}$ \\ ${ }^{1}$ Department of Chemical and Biomolecular Engineering, \\ The University of Melbourne, Australia \\ ${ }^{2}$ Division of Chemical Engineering, \\ The University of Queensland, Australia
}

\begin{abstract}
A modified Volume-of-Fluid numerical method is developed to predict the transient deformation of a viscoelastic drop surrounded by a more viscous Newtonian liquid passing through an axisymmetric microfluidic contraction. Viscoelastic effects are represented using an Oldroyd-B rheological model and can be generated in practice by the addition of small amounts of polymer. The numerical method is tested against experimental observations of viscoelastic drops forming at nozzles. We show that these simulations reliably reproduce flow and drop deformation. Predictions of drop shape and elastic extension are then presented and discussed for drop motion through a microfluidic contraction, and these results are compared against results for an equivalent Newtonian only system.

Keywords: viscoelastic, fluid dynamics, Oldroyd-B, Boger fluid, Volume of Fluid, interfacial, contraction, polymer.
\end{abstract}

\section{Introduction}

Microfluidic technology promises to revolutionise chemical and biological processing in the same way that the integrated circuit revolutionised data processing three decades ago [1]. Key to the operation of microfluidic devices will be the manipulation of droplets of viscoelastic fluids, as many biological and biomedical liquids to be processed contain long chain molecules that stretch and rotate in response to local strain fields. As a contraction can induce mixing in droplets, as well as significantly alter their shape, an understanding of how droplets behave when passing through such a geometry will be essential to the operation of future microfluidic devices. 
Previously published studies concerned with viscoelastic fluids passing through contractions deal with single phase systems. As discussed in Boger [2], elastic contraction flows display fluid behaviour that is markedly different to that of their Newtonian counterparts. The non-linearity of elastic fluids, in particular, makes their use in microfluidic scale devices attractive [1]. While numerical studies of single phase viscoelastic fluids are reaching maturity ([3] for example), numerical studies of viscoelastic systems which contain immiscible fluids are few.

The purpose of this study is threefold. Firstly, we describe how a Volume of Fluid computational algorithm has been modified to simulate viscoelastic immiscible fluid systems, with the elastic stresses simulated using an Oldroyd-B rheological model. We then demonstrate the validity of the algorithm by simulating a multiphase problem for which experimental results are available. After establishing confidence in the technique, we present results for a viscoelastic droplet passing through an axisymmetric contraction, and discuss how the behaviour of this droplet differs from an equivalent Newtonian droplet passing through the same geometry.

\section{Mathematical model}

The system we model consists of two immiscible fluids, one termed the continuous phase and the other the disperse phase. Both phases are viscous and incompressible. Interfacial tension acts at the boundary between the two phases, and the presence of polymers in one or both phases exerts additional elastic stresses on the fluid. We employ the following non-dimensional equations to model the dynamics of this system;

$$
\begin{gathered}
\boldsymbol{\nabla} \cdot \boldsymbol{u}=0 \\
\frac{\partial \phi}{\partial t}+\nabla \cdot \phi \boldsymbol{u}=0 \\
\frac{\partial \rho \boldsymbol{u}}{\partial t}+\nabla \cdot \rho \boldsymbol{u} \boldsymbol{u}=-\boldsymbol{\nabla} p+\frac{1}{\mathrm{Fr}} \rho \hat{\boldsymbol{g}}+\frac{1}{\mathrm{We}} \kappa \hat{\boldsymbol{n}} \delta\left(\boldsymbol{x}-\boldsymbol{x}_{\mathrm{s}}\right)+\frac{1}{\mathrm{Re}} \boldsymbol{\nabla} \cdot \tau \\
\tau=\mu\left[\boldsymbol{\nabla}+(\boldsymbol{\nabla} \boldsymbol{u})^{\top}\right]+\frac{\mu_{\mathrm{p}}}{\mathrm{De}}(\mathrm{A}-\mathrm{I}) \\
\frac{\partial \mathrm{A}}{\partial t}+\boldsymbol{\nabla} \cdot \mathrm{A} \boldsymbol{u}=\mathrm{A} \cdot \boldsymbol{\nabla} \boldsymbol{u}+(\boldsymbol{\nabla} \boldsymbol{u})^{\top} \cdot \mathrm{A}-\frac{1}{\mathrm{De}}(\mathrm{A}-\mathrm{I})
\end{gathered}
$$

Equations (1)-(3) are the continuity, disperse phase transport and momentum equations, respectively. These equations are fairly conventional, with the exception of the third term on the right of eqn. (3) which represents the interfacial tension induced stress jump which occurs across the disperse-continuous phase interface. Equation (4) describes the stress within the fluid resulting from both viscous and elastic contributions, while eqn. (5) describes the evolution of the elastic configuration tensor, $A$.

The above equations are applied in a volume averaged sense when modelling the system. Thus, $\boldsymbol{u}$ represents the fluid velocity, locally volume averaged over 
both phases. Other volume averaged variables include $\phi$, the disperse phase volume fraction, $\rho$, the density, $\mu$, the (solvent only) shear viscosity, and $\mu_{\mathrm{p}}$, the concentration of polymers present, expressed as the increase in shear viscosity of the solution caused by the addition of polymers. The density is calculated from the disperse phase fraction using $\rho=(1-\phi)+\phi \rho_{\mathrm{d}}$ where $\rho_{\mathrm{d}}$ is the non-dimensional disperse phase density. Analogous expressions exist for $\mu$ and $\mu_{\mathrm{p}}$. Note that $\rho, \mu$ and $\mu_{\mathrm{p}}$ are all uniform away from any interface regions. Other variables in the above equations include $\hat{\boldsymbol{g}}$, a unit vector directed in the direction of gravity, $\kappa$, the local curvature of the disperse-continuous phase interface, $\hat{\boldsymbol{n}}$, a unit vector defined along the disperse-continuous phase interface and directed normal to this interface, and $\boldsymbol{x}_{\mathrm{s}}$, the location of the disperse-continuous phase interface.

In non-dimensionalising the equations, velocity has been scaled by $u^{*}$, length by $x^{*}$, density by the continuous phase density $\rho_{\mathrm{c}}^{*}$ and viscosities (including the polymer concentration $\mu_{\mathrm{p}}$ ) by the continuous phase (solvent only) viscosity, $\mu_{\mathrm{c}}^{*}$. These scalings result in three non-dimensional groups describing the ratio between inertial, viscous, gravitational and interfacial forces acting in the system;

$$
\operatorname{Re}=\frac{\rho_{\mathrm{c}}^{*} u^{*} x^{*}}{\mu_{\mathrm{c}}^{*}}, \mathrm{We}=\frac{\rho_{\mathrm{c}}^{*} u^{* 2} x^{*}}{\sigma^{*}} \text { and } \mathrm{Fr}=\frac{u^{* 2}}{g^{*} x^{*}} .
$$

Note that in our notation an asterisk implies a dimensional quantity, a 'c' subscript a continuous phase property, and a ' $d$ ' subscript a disperse phase property.

The Oldroyd-B rheological model has been chosen to represent viscoelastic effects [4]. Oldroyd-B fluids have constant shear viscosities, so are appropriate for modelling Boger fluids such as dilute polymer solutions [2]. In the Oldroyd-B model, polymers are represented as infinitely extensible 'dumbbells', the configuration of which is described by an ensemble averaged tensor $A=<\boldsymbol{R} \boldsymbol{R}>$, where $\boldsymbol{R}$ represents the orientation and length of individual dumbbells. The length of $\boldsymbol{R}$ is normalised so that in the relaxed state, $|\boldsymbol{R}|=1$ and $\mathrm{A}=\mathrm{I}$ (the identity matrix). Equation (5) describes the evolution of $A$. The Deborah number which appears in this equation is the ratio of the relaxation time of the polymer to the timescale of the underlying flow, i.e., De $=t_{\mathrm{p}}{ }^{*} u^{*} / x^{*}$ where $t_{\mathrm{p}}{ }^{*}$ is the polymer relaxation time. Highly elastic fluids have high Deborah numbers, whereas near Newtonian fluids have Deborah numbers close to zero.

\section{Numerical solution technique}

The simulations were performed using a finite volume code originally due to Rudman [5], but modified to account for elastic effects. The finite volume code, minus elastic effects, has been previously used to model the formation and subsequent 'pinch-off' of both Newtonian and generalized Newtonian pendant drops [6], the deformation of Newtonian and shear thinning drops that pass through microfluidic sized axisymmetric contractions [7, 8, 9], and the deformation and breakup of a continuous stream of liquid in a microfluidic 'flow focusing' device [10]. The Volume of Fluid (VOF) technique is used to track the disperse-continuous phase 
interface with the disperse phase volume fraction (the VOF function) advected using a variation of the Youngs scheme [11]. Surface tension forces are applied using a variation of the Continuum Surface Force (CSF) model [12]. The domain is discretised using a uniform, staggered mesh with pressure and volume fractions stored at cell centres, and velocities stored at cell boundaries.

To include elastic effects, we require a solution for the dumbbell configuration tensor A throughout the flow domain. Rather than solve eqn. (5) directly, we have found that for multiphase problems better accuracy is obtained by solving for $\mu_{\mathrm{p}} \mathrm{A}=\mathrm{B}$ instead. In effect, this is solving for the elastic stress field directly rather than the dumbbell configuration field.

To express eqn. (5) in terms of $B$, we first note that as

$$
\mu_{\mathrm{p}}=(1-\phi) \mu_{\mathrm{p}, \mathrm{c}}+\phi \mu_{\mathrm{p}, \mathrm{d}}
$$

( $\mu_{\mathrm{p}, \mathrm{c}}$ and $\mu_{\mathrm{p}, \mathrm{d}}$ are the polymer concentrations in the continuous and disperse phases, respectively), eqn. (2) implies that

$$
\frac{\partial \mu_{\mathrm{p}}}{\partial t}+\nabla \cdot \mu_{\mathrm{p}} \boldsymbol{u}=0
$$

Combining this with eqn. (5) gives the transport equation

$$
\frac{\partial \mathrm{B}}{\partial t}+\nabla \cdot \mathrm{B} \boldsymbol{u}=\mathrm{B} \cdot \boldsymbol{\nabla} \boldsymbol{u}+(\boldsymbol{\nabla} \boldsymbol{u})^{\mathrm{T}} \cdot \mathrm{B}-\frac{1}{\mathrm{De}}\left(\mathrm{B}-\mu_{\mathrm{p}} \mathrm{I}\right)
$$

which is the equation solved for the evolution of B. Elastic stresses are included in the calculation via eqn. (4) once the components of B are known. As the B components are stored at mass cell centres, linear interpolation is used to evaluate any components required at cell vertices.

The main details of the numerical technique used to solve eqn. (8) have been previously described by Davidson and Harrie [13]. For each timestep that the solution is advanced, the technique uses three sequential steps; advection, correction, and the addition of source terms. The correction step ensures that the diagonal components of $\mathrm{B}$ in each computational cell are positive, as is required physically. This technique was originally developed by Singh and Leal [3], however here we perform this correction on the components of $B$ rather than on $A$. The addition of source terms to the evolution of $B$, that is, the addition of all of the terms on the right hand side of eqn. (8) during each timestep, is accomplished using a first order explicit technique that ensures that the determinant of $B$ is positive to first order (in timestep) given that it was positive at the previous timestep [13]. That the determinant of $B$ remains positive is also required physically [3].

The method used to advect the components of B used in this study is new, and differs from that used in [13]. It is motivated by the need to minimise diffusion of $B$ across fluid phase interfaces, while still providing the high order accuracy necessary to reproduce experimentally observed elastic behaviour. 
The advection component of eqn. (8), performed during the advection step, can be represented by

$$
\frac{\partial \mathrm{B}}{\partial t}+\nabla \cdot \mu_{\mathrm{p}} \boldsymbol{u} \mathrm{A}=0 .
$$

The temporal derivative of this equation is discretised using an explicit first order Euler method. In evaluating the spatial derivatives, a value for the flux of $B$, that is $\mu_{\mathrm{p}} \boldsymbol{u} \mathrm{A}$, must be calculated for each computational cell boundary. As the concentration of polymer contained within a computational cell is just a linear combination of the disperse phase volume fraction contained within that cell (see eqn. (6)), the flux of polymer concentration over each boundary $\left(\mu_{\mathrm{p}} \boldsymbol{u}\right)$ can be calculated from the disperse phase volume fraction fluxes $(\phi \boldsymbol{u})$ that are already known from the 'VOF' differencing of the disperse phase transport eqn. (2). The flux of B is calculated by multiplying these polymer concentration fluxes $\left(\mu_{\mathrm{p}} \boldsymbol{u}\right)$ by values of $\mathrm{A}$ approximated at each computational cell boundary and averaged over the timestep duration.

The advantage of using the disperse phase volume fluxes in advecting the $B$ components is that the concentration of polymer within a cell and the elastic stress within that cell are always 'synchronised'. This ensures that in regions where there is no polymer, there will be no advection of elastic stress. It also ensures that no diffusion of elastic stress can occur across an interface between a fluid phase that contains polymer and one that does not, as no flux of polymer occurs across such an interface.

Cell boundary values for the components of A are evaluated using a third order spatially accurate method. This method is based on the QUICK scheme [14], however, the upwind gradients used to evaluate each boundary value are limited to ensure that the diagonal components of the A tensor are positive on each boundary, and that the determinant of $A$ calculated from these boundary values is positive, as required physically. Application of these ideas leads to a temporally first order and spatially second order bounded scheme for the advection of $B$.

\section{Validation: viscoelastic pendant drop}

To demonstrate the validity of the method, we compared published experimental data [15] against simulation results for the formation of a viscoelastic pendant drop in air. Simulation results for this problem were previously presented in [13]. Although the figures shown here were produced using the present version of the code, the results are almost identical to those in [13].

Figure 1 shows selected experimental and simulation images of the pendant drop evolving. The liquid used in the experiments was a water and glycerol mixture containing $0.1 \mathrm{wt} \%$ of $1 \times 10^{6} \mathrm{~g} / \mathrm{mol}$ Polyethylene Oxide (PEO) polymers. The droplet formed on a nozzle of outer radius $2 \mathrm{~mm}$. Using fluid and polymer properties measured by [15], non-dimensional numbers used in the simulations were calculated as $\operatorname{Re}=2.1$, We $=7.5 \times 10^{-4}, \mathrm{Fr}=4.36 \times 10^{-3}$, De $=3.1 \times 10^{-2}$ and $\mu_{\mathrm{p}, \mathrm{d}}=7.06 \times 10^{-1}$, where droplet phase properties were used in the nondimensionalisation. The simulations were performed in axisymmetric coordinates, 


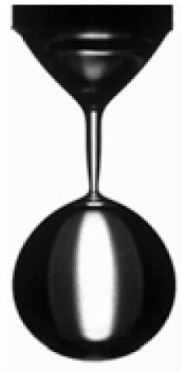

$0 \mathrm{~ms}$

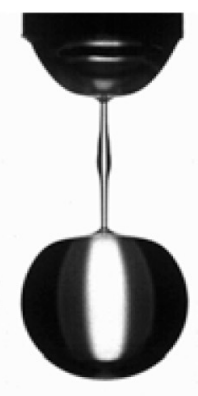

$2 \mathrm{~ms}$

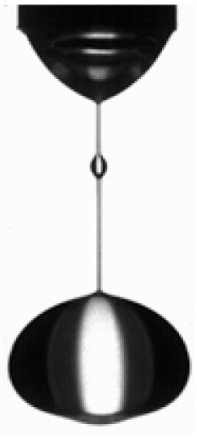

$10 \mathrm{~ms}$

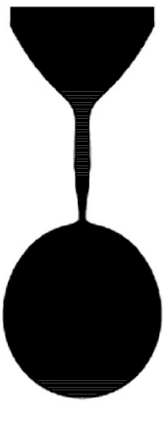

$0 \mathrm{~ms}$

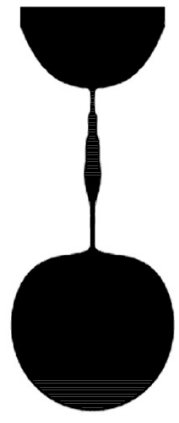

$2 \mathrm{~ms}$

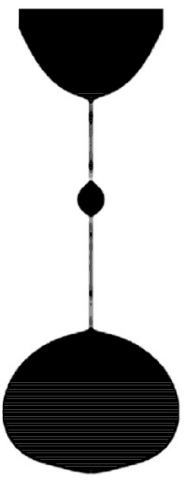

$10 \mathrm{~ms}$

(a) Experiment

(b) Simulation

Figure 1: A comparison of experimental and simulation images for the pendant drop experiment. Relative times between the images are indicated.

using a mesh of $64 \times 288$ cells. Comparing the images of figure 1 shows that the algorithm captures both qualitative and quantitative features of the experiments well. In particular, the necking of the droplet at two positions, which causes the polymers to locally extend, thus preventing breakage and producing the 'beadon-a-string' structure, is captured accurately. Experiments conducted using PEO solutions having different molecular weights and concentrations have also been accurately reproduced [13].

\section{Results: axisymmetric contraction}

The contraction problem consists of a droplet of viscoelastic fluid, entrained in a more viscous continuous phase, and passing through a $4: 1$ axisymmetric contraction. All lengths are non-dimensionalised by the radius of the inlet $x^{*}$ so that the contraction radius is $1 / 4$, the contraction length is 5 and the initial droplet diameter is 1 . Further details of the geometry can be found in the related Newtonian and shear thinning drop deformation studies $[7,8,9]$. The scaling velocity $u^{*}$ is taken to be the average inlet velocity, and gravitational effects are ignored. It is assumed that initially the polymers within the droplet are in a relaxed state so that $A=I$. $A$ computational mesh of $64 \times 768$ cells is used.

Figure 2(a) shows the form of the viscoelastic droplet as it passes through the contraction, as well as the magnitude of the $\sqrt{\operatorname{tr} A}$ that develops within the droplet: $\sqrt{\operatorname{tr}(A)}$ is a measure of the average length of the polymers. The parameters chosen for the simulation could represent a droplet of water and glycerol based dilute PEO solution, similar to that used in the pendant drop experiments of figure 1, entrained in a low viscosity Silicon oil, and passing through a $x^{*}=100 \mu \mathrm{m}$ contraction with an average continuous phase inlet velocity of $u^{*}=3 \mathrm{~cm} / \mathrm{s}$. This experimental 


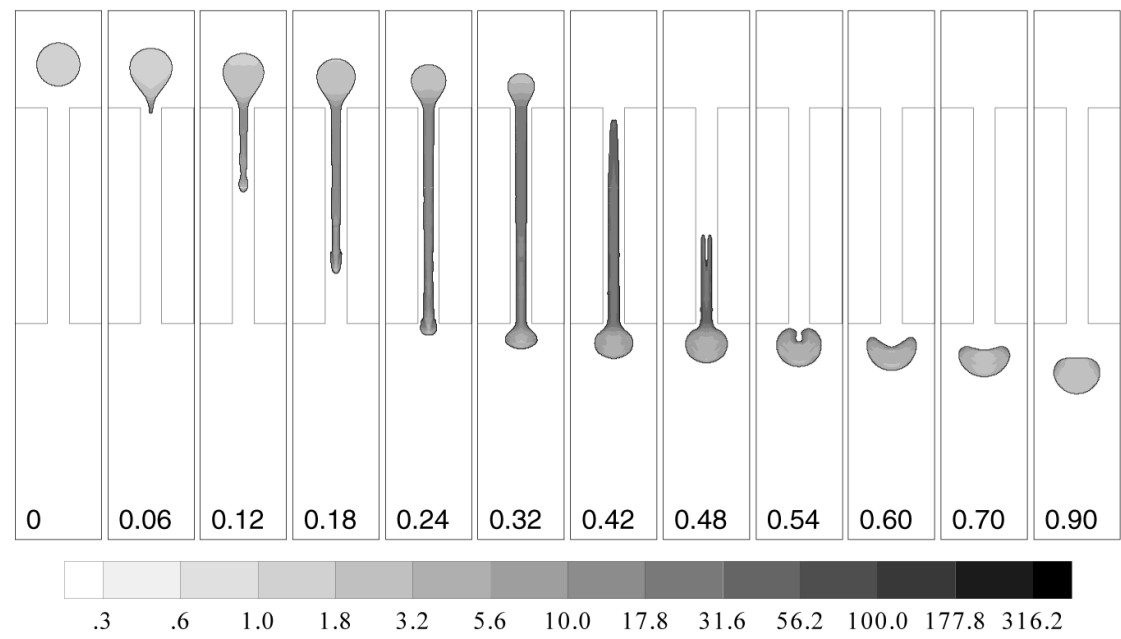

(a) Viscoelastic droplet with $\mathrm{De}=0.18$ and $\mu_{\mathrm{p}, \mathrm{d}}=1$.

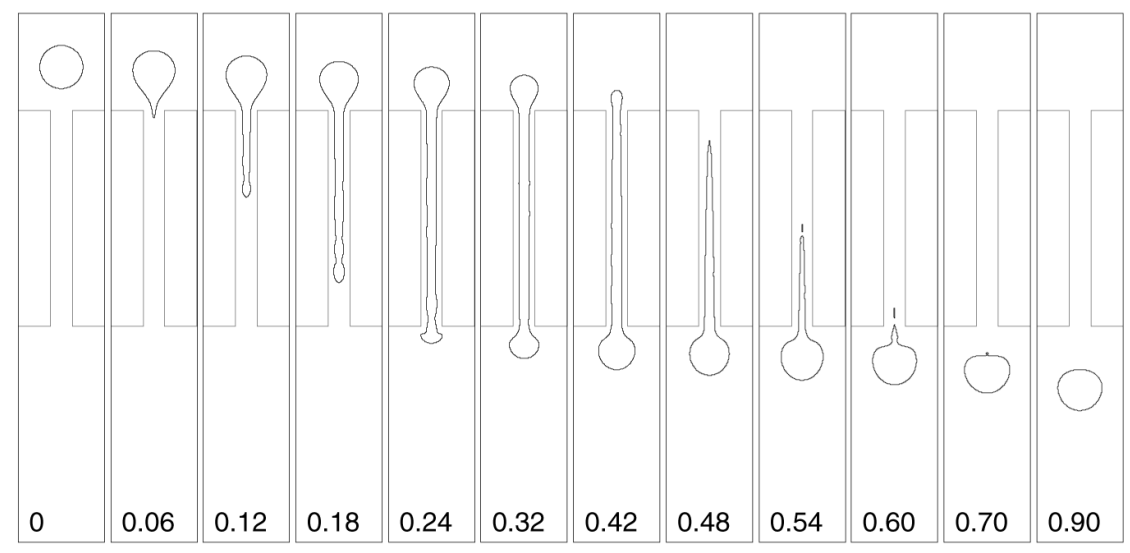

(b) Equivalent Newtonian droplet.

Figure 2: Images showing how viscoelastic and Newtonian droplets deform as they pass through the $4: 1$ axisymmetric contraction. For both cases $\mathrm{Re}=$ 0.1 , We $=9.09 \times 10^{-3}, \mu_{\mathrm{d}}=0.1$ and $\rho_{\mathrm{d}}=1$. Each frame is annotated with its non-dimensional time, and the shading in the viscoelastic case represents $\sqrt{\operatorname{tr}(A)}$, a measure of the average polymer extension.

setup is feasible with current microfluidic technology. Figure 2(b) shows how an identical droplet to that of figure 2(a) would behave in the same system if the droplet contained no polymers.

The first five frames of figures 2(a) and 2(b) show the droplets entering and moving through the contraction. In the Newtonian case, the axial acceleration of 
the continuous phase near the entrance to the contraction deforms the drop into an inverted 'tear' shape, with a narrow point at its leading tip. By $t=0.012$, this tip has rounded, as both interfacial tension and drag from the surrounding continuous phase pull the tip back towards the main body of the droplet. By $t=0.18$, this tip has become quite bulbous, with secondary interfacial waves propagating back along the extended filament towards the contraction entrance. This process of 'tip bulbing' has been observed in low viscosity Newtonian droplet contraction simulations [9].

The viscoelastic droplet behaves very similarly as it enters the contraction, but the further it progresses into the contraction, the more the polymers extend and alter its behaviour. At the entrance to the contraction, the extensional strain that the polymers experience as the fluid accelerates extends the polymers in the axial direction. This extension causes a small axial stress on the droplet, which 'blunts' the sharpness of the droplet tip at $t=0.06$. As the droplet continues into the contraction, shear stresses, exerted by the more viscous continuous phase, extend the polymers more significantly, and transport them around the droplet. Polymer extension occurs mainly in two areas; at the leading tip, where the droplet experiences considerable extensional strain rates, and along the sides of the filament, where the droplet experiences large shear strains.

Noticeable effects of polymer extension on droplet deformation do not appear until around $t=0.18$. At this time, the distribution of $\sqrt{\operatorname{tr}(\mathrm{A})}$ within the leading bulb of the droplet has becomes quite significant and complex, with maximum values of $\sqrt{\operatorname{tr}(A)}$ in this region of 40 and greater. The resulting elastic stresses cause the leading tip bulb to be more 'arrow' shaped than the Newtonian bulb, and also dampen the interfacial waves that were observed on the Newtonian droplet at this time. By $t=0.24$, maximum $\sqrt{\operatorname{tr}(\mathrm{A})}$ values within the droplet have grown to 50 . These extensions are located just behind the leading bulb of the droplet, along its interface.

At times between $t=0.32$ and $t=0.6$, the behaviour of the droplets differs mainly in the way in which rear of the each deforms. In the Newtonian case, the rear tip of the droplet forms a fine point, from which a small amount of fluid is shed. Fluid is shed from this tip as drag from the continuous phase, which is directed towards the contraction entrance, has a greater magnitude than interfacial tension, which pulls the tip towards the contraction exit. This behaviour has been observed in previous low viscosity Newtonian droplet deformation simulations [9].

The rear tip of the viscoelastic droplet behaves quite differently, instead forming a distinctive forked tail which leaves the contraction earlier than that of the Newtonian droplet. To understand why, we note that during these later times, the shear stresses that act on the droplet interface affect the polymers in two ways: Firstly, large shear rates caused by the interfacial shear stress extend any polymers that lie close to the interface but within the droplet fluid. These polymers are orientated in a direction that is almost tangential to the interface. A close examination of figure 2(a) at $t=0.32$ for example shows that near the interface of the droplet, $\sqrt{\operatorname{tr}(A)}$ is higher than in the body of the droplet, and can reach values as large as 70 near the exit to the contraction. Secondly, the interfacial shear stress moves 
droplet fluid that is adjacent to the interface backwards relative to the leading tip of the droplet, that is, towards the rear of the droplet. Thus, polymers that are within the droplet and adjacent to the interface are extended and moved towards the rear of the droplet as it progresses through the contraction.

At the rear tip, these extended polymers exert stresses on the fluid, changing the shape of the rear interface. When the rear of the droplet first enters the contraction, its shape is almost pointed. As the tip moves into the contraction however, polymers at the tip become extended and orientated parallel to the droplet interface. At the very end of the droplet, these polymers are directed slightly inwards, as the interface shape here is directed towards a single point. As these polymers are in tension, they exert an elastic stress on the fluid, which pulls the centre of the rear tip forwards, creating the inverted 'dimple' at the rear of the droplet observed at $t=0.32$. As the flow of polymers along the droplet interface and towards the rear of the droplet continues, the dimple grows, and the forked tail that is shown at $t=0.48$ in figure 2(a) develops. The growth of this tail is reinforced by the high centreline velocity of the continuous phase that follows the droplet through the contraction. The viscoelastic droplet exits the contraction sooner than the Newtonian droplet simply because its tail is blunt, so is shorter than the narrower Newtonian one.

Beyond the contractions, both droplets shorten and expand radially as the surrounding continuous phase fluid decelerates. The simulations predict that the fluid shed from the rear of the Newtonian droplet coalesces with the rest of the droplet at $t \approx 0.72$. As discussed in Harvie et al. [9] however, the timing of this behaviour may or may not be physically realistic as the film drainage that occurs between these two droplets as they coalesce is not captured by the resolution of the computational mesh. At times beyond $t=0.7$, interfacial tension quickly reforms the Newtonian droplet into an approximately spherical steady state form.

In the viscoelastic case, the forked tail that was present on the droplet while it was within the contraction shortens and expands radially, forming the bulbous ' $U$ ' shape observed at $t=0.54$ in figure 2(a). The droplet then moves towards a more spherical shape under the action of interfacial tension, however, as the polymers take some time to relax, the viscoelastic droplet takes longer to reach a steady state form than the Newtonian droplet does. Even at $t=0.9$, a time at which the Newtonian droplet is almost spherical, the viscoelastic droplet still has a 'flattened' top, with polymers within it having lengths of up to 9 . Simulations show that the viscoelastic droplet does not reach its steady and effectively relaxed elastic state until about $t=1.6$.

\section{Acknowledgement}

This research was supported by the Australian Research Council Grants Scheme.

\section{References}

[1] Squires, T.M. \& Quake, S.R., Microfluidics: fluid physics at the nanoliter scale. Reviews of Modern Physics, 77(3), pp. 977-1026, 2005. 
[2] Boger, D.V., Viscoelastic flows through contractions. Annual Review of Fluid Mechanics, 19, pp. 157-182, 1987.

[3] Singh, P. \& Leal, L.G., Finite-element simulation of the start-up problem for a viscoelastic fluid in an eccentric rotating cylinder geometry using a thirdorder upwind scheme. Theoretical and Computational Fluid Dynamics, 5, pp. 107-137, 1993.

[4] Oldroyd, J.G., On the formulation of rheological equations of state. Proceedings of the Royal Society of London, A, 200(1063), pp. 523-541, 1950.

[5] Rudman, M., A volume-tracking method for incompressible multifluid flows with large density variations. International Journal for Numerical Methods in Fluids, 28, pp. 357-378, 1998.

[6] Davidson, M.R. \& Cooper-White, J.J., Pendant drop formation of shearthinning and yield stress fluids. Applied Mathematical Modelling, 2005. Accepted.

[7] Harvie, D.J.E., Davidson, M.R. \& Cooper-White, J.J., Simulating the deformation of newtonian and non-newtonian drops through a micro-fluidic contraction. 15th Australasian Fluid Mechanics Conference, University of Sydney: NSW, Australia, 2004.

[8] Harvie, D.J.E., Davidson, M.R., Cooper-White, J.J. \& Rudman, M.J., A numerical parametric study of droplet deformation through a microfluidic contraction. ANZIAM J, 46(E), pp. C150-C166, 2005.

[9] Harvie, D.J.E., Davidson, M.R., Cooper-White, J.J. \& Rudman, M.J., A parametric study of droplet deformation through a microfluidic contraction: Low viscosity Newtonian fluids. Chemical Engineering Science, 2005. Submitted.

[10] Davidson, M.R., Harvie, D.J.E. \& Cooper-White, J.J., Flow focusing in microchannels. ANZIAM J, 46(E), pp. C47-C58, 2005.

[11] Youngs, D.L., Time-dependent multimaterial flow with large fluid distortion. Numerical Methods for Fluid Dynamics, eds. K. Morton \& M. Baines, Academic Press, pp. 273-285, 1982.

[12] Brackbill, J.U., Kothe, D.B. \& Zemach, C., A continuum method for modelling surface tension. Journal of Computational Physics, 100, pp. 335-354, 1992.

[13] Davidson, M.R. \& Harvie, D.J.E., Simulations of pendant drop formation of a viscoelastic liquid. Korea-Australia Rhoelogy Journal, 2005. Submitted.

[14] Leonard, B.P., A stable and accurate convective modelling procedure based on quadratic upstream interpolation. Computer Methods in Applied Mechanics and Engineering, 19, pp. 59-98, 1979.

[15] Tirtaatmadja, V., McKinley, G.H. \& Cooper-White, J.J., Drop formation and breakup of low viscosity elastic fluids: Effects of molecular weight and concentration. Journal of Non-Newtonian Fluid Mechanics, 2005. Accepted. 